\title{
Designing Systems to Augment Social Interactions
}

\section{Ionut Damian}

University of Augsburg

Elisabeth André

University of Augsburg

\section{Editor:}

Albrecht Schmidt

albrecht.schmidt@ifi.lmu.de

\author{
Thanks to the rapid advancement of mobile and \\ wearable technologies over the past decade, a new \\ shift in social skills training lies ahead that will allow \\ users to continuously monitor and improve their social \\ behavior during actual social interactions. At the \\ same time, despite the demonstrated effectiveness of \\ social augmentation, researchers must address
}

several limitations.

Social behavior lies at the very core of being human. We engage in social interactions multiple times every day. For example, we speak with friends, buy products from a salesperson, or hold conversations with colleagues. Yet, some types of social interactions - speaking in public, being interviewed, participating in a negotiation, and so on - often seem to be governed by a special set of rules that many of us struggle with. However, these kinds of interactions are the ones in which the outcomes are the most crucial. For example, a job interview can decide a person's employment status, or a presentation in school might have a large impact on a student's final grade. The problem is amplified by the fact that in such critical situations stress can make our bodies go into "auto pilot" mode, rendering our cognitive minds oblivious to our body's use of gestures, postures, or even speech.

Imagine a computer system able to monitor our behavior during social interactions and give us subtle feedback on how to improve. For example, when speaking in public, such a system could help us maintain eye contact with the audience or control our speaking rate by informing us if we are too slow and boring, or are too fast and unintelligible. The same system could also help us make a better impression during job interviews by correcting our body posture and use of gestures. Moreover, persons who suffer from various disabilities, such as autism or Parkinson's disease, could use the system to better regulate their behavior, thus avoiding misunderstandings and generally increasing their functional independence. Such a system would effectively "augment" the user's social skills. 
These social augmentation systems can be seen as an extension of Douglas Engelbart's framework for augmenting human intellectl and the personal augmentation concepts of Cassandra Xia and Pattie Maes. ${ }^{2}$ Whereas they focused on problem solving, memory, decision making, motivation and mood, we focus on a different yet equally important domain of human intellect: social behavior.

\section{DESIGN REQUIREMENTS}

Since social augmentation systems are meant to be used during real social interactions, a careful design of the system is paramount. To get a grip on this issue, we devised a set of requirements for the design of social augmentation systems. These have been informed by both literature and empirical research. ${ }^{3}$

First, the user should be able to correctly perceive and process the information delivered by the social augmentation. From a psychological point of view, the augmentation represents a secondary task for the user whereas the social interaction is the primary task. Considering this, the first requirement can be formulated as follows: The social augmentation must be able to momentarily draw enough attention from the primary task to allow information from a social augmentation task to be perceived and processed.

However, it is crucial that the augmentation not draw too much attention lest it distract the user and disrupt the social interaction. According to distributive attention models, ${ }^{4-5}$ tasks can be carried out in parallel without quality degradation as long as enough processing resources are available. Thus, to reduce the amount of distraction, the social augmentation needs to be economical with its demand of resources.

Yet, if the social augmentation is to guide the user to a more desirable behavioral state, it must first be able to generally elicit a change in behavior. Thus, the provided information must be understandable, sufficiently detailed, and relevant to both the user and the moment in which it is delivered. Informing a user who, say, is making a presentation that he or she talked too loudly five minutes ago is not only irrelevant but also confusing. This leads us to the third requirement: The provided information must be appropriate for facilitating the intended change in behavior.

So far, the social augmentation can trigger a change in user behavior without much disturbance. What is still missing is the relation to the social interaction mentioned earlier. Specifically, it is important that the augmentation does not just trigger any change in behavior, but one that contributes to the goals of the user in the interaction. For example, in a job interview, the augmentation should help the user make a better impression and thus increase his or her chances of employment.

The social aspect of the augmentation means that the physical form and aspect of the system is also critical. More specifically, the augmentation system should not hinder verbal or nonverbal communication within the social interaction, nor should it break social conventions or otherwise disrupt that interaction. For example, the use of head-mounted displays (HMDs) might prevent perception of the user's gaze signals, interfering with one critical communication channel. Moreover, the augmentation must be mindful of its impact not only on the user, but also on the persons with whom the user is interacting.

Finally, privacy and transparency concerns also need to be addressed. Throughout history, many promising technologies have encountered resistance over such concerns. When the first truly mobile camera, the Kodak box camera, appeared in 1888, it was heavily criticized and even forbidden in certain public places. Thus, the delicate handling of privacy issues is crucial. One approach is to ensure users understand that social augmentation will entail the loss of some privacy. According to Jason Hong, ${ }^{6}$ users will accept this if the perceived value of the system 
matches or exceeds that of the lost privacy. It is thus paramount that the social augmentation respects the privacy of both users and bystanders.

\section{BEHAVIORAL FEEDBACK LOOP}

In previous work, ${ }^{7,3}$ we introduced the behavioral feedback loop as the driving force behind social augmentation. In simple terms, a feedback loop occurs when the output of a system is repeatedly and continuously fed back to the system as input, thus forming a closed loop. From the point of view of social augmentation, feedback loops are particularly interesting due to their self-regulating nature. Thus, the goal of generating self-awareness discussed earlier can be directly translated to a feedback loop structure. The user's behavior (output) is recorded and fed back to the user (input) continuously, generating awareness of one's own behavior. For this, physical artifacts, such as miniaturized sensors or lightweight displays, are used to both perceive the user's behavior and deliver real-time feedback. Now, through intelligent and goal-oriented manipulation of the feedback loop, the user's behavior can be steered toward a more beneficial state for the social interaction. Figure 1 illustrates the resulting two-step pipeline of a behavioral feedback loop: The user's behavior is first analyzed in real time and then, based on its quality, feedback is automatically generated and delivered to the user.

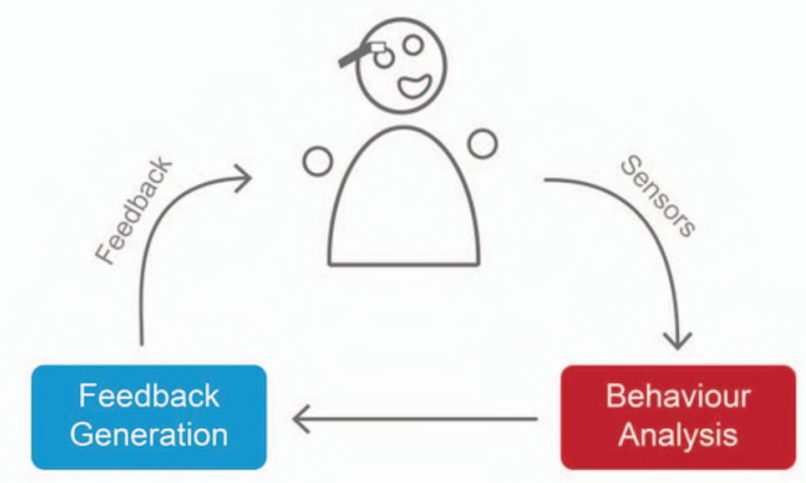

Figure 1.The behavioral feedback loop. The user's behavior is first analyzed in real time and then, based on its quality, feedback is automatically generated and delivered to the user.

\section{EXAMPLES}

Social augmentation systems can be deployed in different scenarios to help users improve the outcomes of their interactions or overcome certain disabilities. Here we provide three concrete use cases for social augmentation.

\section{Augmenting Public Speaking}

Public speaking is a distinct type of social interaction that requires speakers to deliver an informative message to their audience while entertaining and inspiring enthusiasm at the same time. This makes speaking in public a particularly stressful experience. The Logue ${ }^{8}$ social augmentation system, shown in Figure 2, attempts to relieve some of this pressure by delivering direct and objective feedback on the quality of one's speech rate, body energy and openness, as well as providing instructions on how to improve it. To achieve this, social-signal processing techniques are employed to analyze the speaker's performance using data from a microphone and a depth camera. Based on this analysis, feedback is generated and delivered to the user unobtrusively in real time using an HMD. 


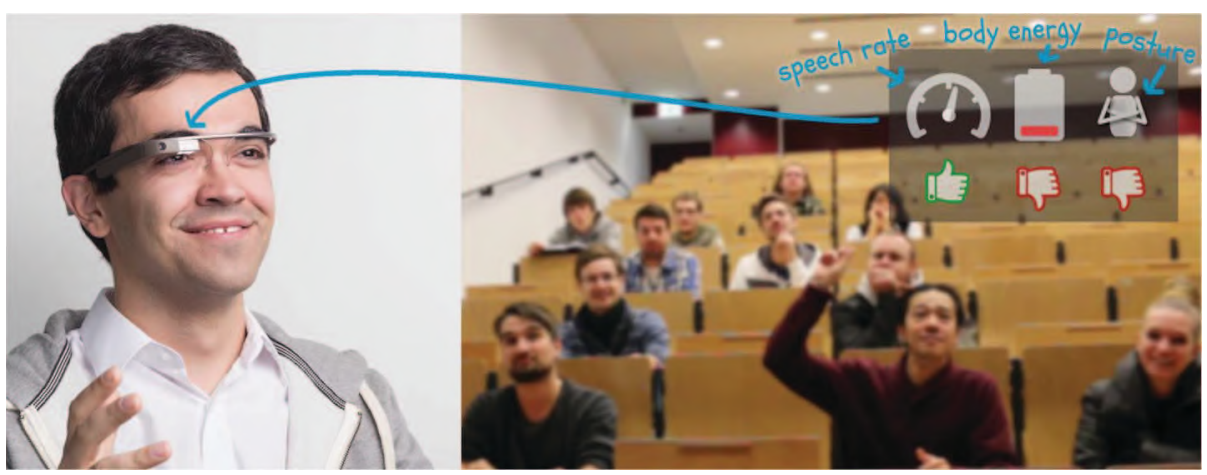

Figure 2. The Logue social augmentation system delivers live visual feedback to a person speaking in public via a head-mounted display.

\section{Augmenting Group Discussions}

Unlike speaking in public, where conversation is mostly one sided and exchanges between speaker and audience are limited, traditional face-to-face interactions are more susceptible to disturbances as they contain complex exchanges of verbal and nonverbal messages that are governed by a delicate set of unwritten rules. We designed an augmentation system that attempts to overcome this problem by providing feedback using different modalities. The system helps the user control their speaking time during group discussions by providing auditory, tactile, or visual feedback in real time. ${ }^{7}$

\section{Augmenting the Speech of Adults with Disabilities}

One common dysfunction associated with autism is atypical prosody. ${ }^{9}$ It impacts the rate, loudness, and pitch of the speaker's voice, potentially leading to misunderstandings during social interactions. Louanne Boyd and her team ${ }^{10}$ developed a social augmentation system that targets adults with autism. Using Google Glass, their system provides visual feedback whenever the user exhibits an atypical vocal pitch or loudness.

A similar approach was proposed by Roisin McNaney and her colleagues ${ }^{11}$ for helping people with Parkinson's, who often "have an impaired perception of how loud they are speaking," better regulate the loudness of their voice. Their LApp system uses Google Glass to continuously monitor the loudness of the user's voice and gives visual feedback whenever it drops below a predefined threshold.

\section{CONCLUSION}

Social skills training has evolved over the past few decades from using manual and analog forms of knowledge transfer to intelligent virtual simulation environments, which can automatically react and adapt to the learner. Now, thanks to the rapid advancement of mobile and wearable technologies over the past decade, a new shift in social skills training lies ahead that will allow users to continuously monitor and improve their social behavior during actual social interactions.

Despite the demonstrated effectiveness of social augmentation, researchers must address several limitations. As with all types of augmentation, there is a risk of the user learning to rely on the system too much. This could make the user dependent on the system and thus unable to act without it. Similarly, the user might focus too much on "pleasing" the system and ignore the real world-for example, cues from interlocutors. This could result in abnormal behavior because social augmentation is meant to help users improve their current social behavior, not be a substitute for it. Nevertheless, intelligent feedback design and advanced behavior analysis routines could be used proactively to minimize such risks. 


\section{REFERENCES}

1. D.C. Engelbart, Augmenting Human Intellect: A Conceptual Framework, government report AFOSR-3223, Stanford Research Institute, 1962; www.dougengelbart.org/pubs/papers/scanned/Doug_EngelbartAugmentingHumanIntellect.pdf.

2. C. Xia and P. Maes, "The Design of Artifacts for Augmenting Intellect," Proc. 4th Augmented Human Int'l Conf. (AH), 2013, pp. 154-161.

3. I. Damian, Social Augmentation Using Behavioural Feedback Loops, thesis, Universität Augsburg, 2017; opus.bibliothek.uniaugsburg.de/opus4/frontdoor/index/index/docId/37750.

4. D. Navon, "Resources-A Theoretical Soup Stone?," Psychological Rev., vol. 91, no. 2, 1984, pp. 216-234.

5. C.D. Wickens, "Multiple Resources and Performance Prediction," Theoretical Issues in Ergonomics Science, vol. 3, no. 2, 2002, pp. 159-177.

6. J. Hong, "Considering Privacy Issues in the Context of Google Glass," ACM Comm., w3c recommendation, vol. 56, no. 11, 2013, pp. 10-11.

7. I. Damian, T. Baur, and E Andre, "Measuring the Impact of Behavioural Feedback Loops on Social Interactions," Proc. 18th ACM Int'l Conf. Multimodal Interaction (ICMI), 2016, pp. 201-208.

8. I. Damian et al., "Augmenting Social Interactions: Realtime Behavioural Feedback using Social Signal Processing Techniques," Proc. 33rd Ann. ACM Conf. Human Factors in Computing Systems (CHI), 2015, pp. 565-574.

9. L. Kanner, "Autistic Disturbances of Affective Contact," Acta Paedopsychiatrica, vol. 35, no. 4, 1968, pp. 100-136.

10. L.E. Boyd et al., "SayWAT: Augmenting Face-to-Face Conversations for Adults with Autism," Proc. 2016 CHI Conf. Human Factors in Computing Systems (CHI), 2016, pp. 4872-4883.

11. R. McNaney et al., "LApp: A Speech Loudness Application for People with Parkinson's on Google Glass," Proc. 33rd Ann. ACM Conf. Human Factors in Computing Systems (CHI), 2015, pp. 497-500.

\section{ABOUT THE AUTHORS}

Ionut Damian is a postdoctoral researcher at the Institute of Computer Science, University of Augsburg. Contact him at damian@hcm-lab.de.

Elisabeth André is a full professor of computer science and chair of Human-Centered Multimedia in the Faculty of Applied Informatics at the Institute of Computer Science, University of Augsburg. Contact her at andre@hcm-lab.de. 